\title{
Multi-Environment Analyses of Genetic Components and Combining Abilities in Relation to Heterosis in Okra [Abelmoschus esculentus (L.) Moench]
}

\author{
Sanket J. More $^{1 *}$, K.N. Chaudhari ${ }^{2}$, G.B. Vaidya ${ }^{3}$ and S.L. Chawla ${ }^{2}$ \\ ${ }^{1}$ ICAR-Central Tuber Crops Research Institute, Sreekariyam PO, Thiruvananthapuram, \\ Kerala 695 017, India \\ ${ }^{2}$ ASPEE College of Horticulture and Forestry, Navsari Agricultural University, Navsari, \\ Gujarat 396 450, India \\ ${ }^{3}$ ANKUR Seed Co. PVT. LTD., Nagpur, India \\ *Corresponding author
}

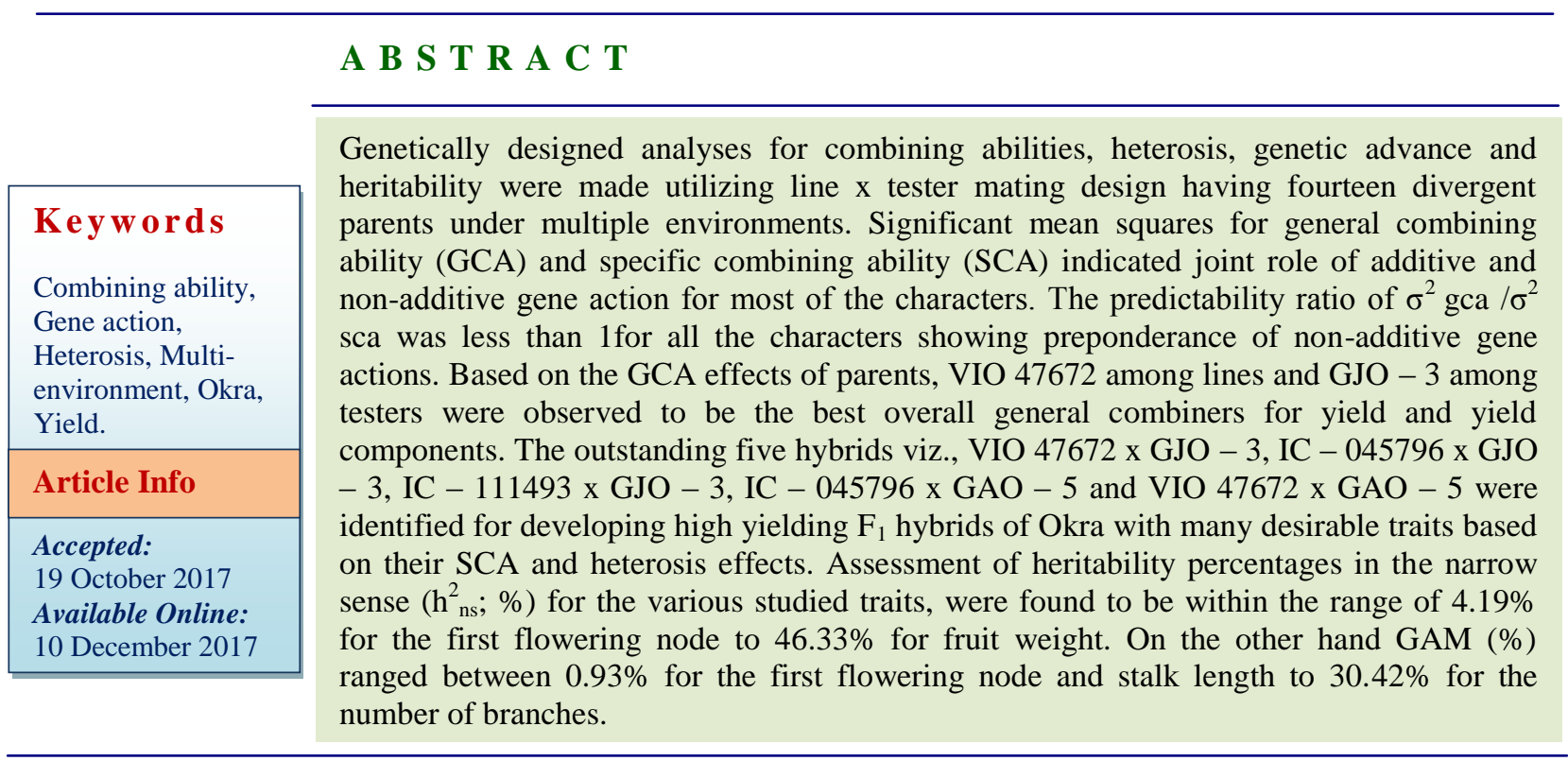

\section{Introduction}

Okra (Abelmoschus esculentus (L.) Moench) is an economically important vegetable crop grown in tropical and sub-tropical parts of the world. India is a major okra producing country in the world comprising of $72 \%$ of total area under okra (FAOSTAT, 2013). Selection of suitable parents is an important step for enhancement of any breeding program for crop varietal improvement (Solankey et al., 2016). The knowledge of nature of gene action governing the expression of various traits could be helpful in predicting the effectiveness of selection (Dabhi et al., 2010). The combining ability is the important genetic tool, which provides a guideline for an assessment of the relative breeding potential of the parents or identifying the best combiners, which may be hybridized either to exploit heterosis or to accumulate fixable genes (Katagi et al., 
2015). Exploitation of heterosis in okra has been recognized as a practical tool in providing the breeders a means of improving yield and other important traits (Lyngdoh et al., 2013; Singh et al., 2016). The success of a breeding programme is determined by useful gene combinations in the form of high combining inbred (Obiadalla et al., 2013). Several researchers have reported the presence of heterosis for fruit yield in okra (Akotkar et al., 2014; Badiger et al., 2014; Katagi et al., 2015; Kumar et al., 2014; Flemine Xavier et al., 2016; Maciel et al., 2017). Several high yielding hybrids have been developed by Indian Institute of Vegetable Research (ICAR-IIVR), Varanasi, India among which "Kashi Bhairav" has achieved yield up to 20-22 MT/ha. Average productivity of okra in India is $11.35 \mathrm{MT} / \mathrm{ha}$ (Anonymous, 2016). A large potential yield gap exists between farmers' yield and than that of documented by various research organizations. The major problem in okra cultivation is lack of high-yielding varieties/hybrids (Solankey et al., 2016). The productivity of okra should be increased through hybridization and recombination. Yield barriers can be mitigated by planning effective breeding program involving best suitable combiner parents to develop high yielding hybrids. In this connection present study was taken to obtain clear and determined information about the relative importance of gene actions involved in the inheritance of some growth and yield traits of okra through the estimation of both the general and specific combining abilities in relation to heterosis over multiple environments using a line $\mathrm{x}$ tester approach.

\section{Materials and Methods}

The study was conducted between September, 2013 and February, 2015 (including crossing programme during September to December, 2013). The experiment consisting 55 genotypes including 14 parents their 40 hybrids and one standard check, was laid out in a randomized complete block design (RBD) with three replications over three environments (Table 1) at the Regional Horticultural Research Station, ASPEE College of Horticulture and Forestry, Navsari Agricultural University, Navsari, situated at coastal region of South Gujarat. Geographically, it is situated at $20^{\circ}-37^{\prime} \mathrm{N}$ latitude and $72^{\circ}-54^{\prime} \mathrm{E}$ longitude with an altitude of 11.98 meters above the Mean Sea Level. All recommended horticultural practices along with plant protection measures were followed uniformly and timely.

\section{Parental material and recorded data}

Fourteen diverse parents (Table 2) were crossed to produce forty $F_{1}$ hybrids. Selfed truthful seeds treated with Thiram $4 \mathrm{~g} / \mathrm{kg}$ of seeds were sown (10 plants/row) in line $\mathrm{x}$ tester fashion at spacing of $60 \times 45 \mathrm{~cm}$ in. Observations on thirteen traits were recorded. The crude fiber content from okra fruit was determined by the method as illustrated by Thimmaiah (1999).

\section{Statistical analysis}

The data were statistically analyzed, using Windostat version 8.6. The differences among the means of various yield components were tested, using Duncan's Multiple Range Test (L.S.R.) at 0.05 level of probability.

\section{Analysis of combing ability}

The combining ability analysis was carried out as per the method given by Kempthorne (1957). The critical differences were calculated as advocated by Panse and Sukhatme (1967). The heritability percentage estimation and categorization (low, moderate and high) and narrow sense heritability estimation were calculated as suggested by Robinson et al., (1955). Genetic advance in absolute unit (GA) and percent of the mean 
(GAM at 5\%), summing selection of superior $5 \%$ of the genotypes were estimated as the method illustrated by Jhonson et al., (1955).

\section{Results and Discussion}

\section{Analysis of variance of combing ability}

The analysis of variance depicting mean squares is presented in Table 3. Pooled analysis of variance over environments revealed that all the three environments were different as estimates among them were found significant for all the traits. The differences among hybrids were highly significant for all the traits in individual and across the environments. The variance due to parents was further partitioned into variance due to interaction of females and males. The results revealed that mean squares due to males $\mathrm{x}$ females were highly significant for all traits across environments. The variance due to hybrids was highly significant for all the traits over the environments. The interactions of hybrids $\mathrm{x}$ environments and Female $\mathrm{x}$ Male $\mathrm{x}$ Environment were significant for all the traits except for the number of branches and fruit diameter which revealed that the hybrids, males and females were sensitive to the environments. In pooled analysis, significant environmental variances $\left(\sigma^{2}\right.$ e) for all the traits not only signified the appreciable influence of environments on the expression of traits but also confirmed the fact that considerable differences existed among the environments under which the study was conducted.

\section{Genetical analysis of various components}

The results of genetical analysis of various components are presented in Table 4. The results illustrated that the estimated variances for the effects of both general and specific combining abilities ( $\sigma^{2} \mathrm{gca} / \sigma^{2}$ sca) showed relatively high values for all the studied characters. Moreover, it was noticed for all the characters that non-additive gene effects were found to be more pronounced for their contributions to the genetic variability. The estimates of $\mathrm{h}^{2}{ }_{\mathrm{ns}} \%$ showed relatively low or low percentages for the all the characters except number of branches $(31.46 \%)$, fruit diameter $(39.47 \%)$ and fruit weight $(46.33 \%)$. Similarly, the estimations of genetic advance as per cent mean, for the various characters studied, were found to be within the range of $0.93 \%$ for first flowering node to $30.42 \%$ for number of branches. All the characters studied exhibited relatively low GAM except number of branches (30.42\%). In present investigation, none of the characters studied exhibited high heritability coupled with high genetic advance. The character number of braches exhibited moderate heritability coupled with high genetic advance. Significant results of various components indicated that males-females and parentshybrids interacted significantly with each other. These results are in agreement with those of Das et al., (2013) and Badiger et al., (2014).

\section{General and specific combining abilities} estimates in relation to heterosis

The estimates of combining abilities (Table 5 and 6) showed that among lines, IC 045796 and VIO 47672 were found to be the most suitable combiners for most of the traits. Among testers GJO-3 was found to be the best combiners for most of the characters. Hybrids JOL-08-13 x Arka Anamika and VIO-44244 x Varsha Uphar registered highest significant SCA effects for earliness traits like days to first flowering (-1.98) and first flowering node (-0.53), respectively. Regarding plant growth characters like for number of branches and plant height VIO 44244 x Varsha Uphar (0.29) and VIO 47672 $\mathrm{x}$ GJO - 3 (18.28), registered highest significant SCA effects respectively. 
Table.1 Details of environments taken under study

$$
\text { Environments }
$$

$\mathrm{E}_{1} \quad$ Summer, 2014 (February to May, 2014)

$\mathrm{E}_{2} \quad$ Rainy, 2014 (June to September, 2014)

$\mathrm{E}_{3} \quad$ Winter, $2014-15$ (November, 2014 to February, 2015)

Table.2 Parental material used for the study

\begin{tabular}{ll}
\hline Parent & Source/Origin \\
\hline Lines & \\
\hline VIO 44244 & AVRDC, Taiwan \\
IC -11493 & NBPGR, New Delhi, India \\
JOL $-08-13$ & Junagadh Agricultural University, Junagadh (Gujarat) \\
EC -284327 & NBPGR, New Delhi, India \\
IC -045796 & NBPGR, New Delhi, India \\
IC -052273 & Junagadh Agricultural University, Junagadh (Gujarat) \\
JOL $-10-18$ & Junagadh Agricultural University, Junagadh (Gujarat) \\
AOL $-09-17$ & Anand Agricultural University, Anand (Gujarat) \\
VIO 47672 & AVRDC, Taiwan \\
EC -305623 & NBPGR, New Delhi, India \\
\hline Testers & \\
\hline GAO -5 & Anand Agricultural University, Anand (Gujarat) \\
GJO -3 & Junagadh Agricultural University, Junagadh (Gujarat) \\
Arka Anamika & IIHR, Bengaluru, India \\
Varsha Uphar & IIHR, Bengaluru, India \\
\hline Commercial check: Sonakshi (Hybrid; Nunhems Company) \\
\hline
\end{tabular}

Table.3 Analysis of variance for the yield components of the fourteen parents and their forty $\mathrm{F}_{1}$ hybrids of okra across the environments

\begin{tabular}{|c|c|c|c|c|c|c|c|}
\hline Source & Environments & Hybrids & $\begin{array}{l}\text { Females } \\
\text { (F) }\end{array}$ & $\begin{array}{l}\text { Males } \\
(\mathrm{M})\end{array}$ & $\begin{array}{l}\text { Females x } \\
\text { Male(F x } \\
\text { M) }\end{array}$ & $\begin{array}{l}\text { Hybrids x } \\
\text { Environnements }\end{array}$ & $\begin{array}{l}\text { (Female x } \\
\text { Male) x } \\
\text { Environments }\end{array}$ \\
\hline D.F. & 2 & 39 & 9 & 3 & 27 & 78 & 54 \\
\hline \multicolumn{8}{|l|}{ Traits } \\
\hline DFF & $1272.9^{* *}$ & $27.33^{* * *}$ & 43.99 & 28.92 & $21.61^{* *}$ & $13.24^{* * *}$ & $14.50^{* *}$ \\
\hline FFN & $118.4^{* *}$ & $0.95^{* *}$ & 0.77 & 0.880 & $1.02^{* *}$ & $1.02^{* * *}$ & $0.88^{* *}$ \\
\hline IL & $1279.5^{* *}$ & $5.08^{* *}$ & 6.04 & 10.58 & $4.16^{* *}$ & $2.59^{* *}$ & $2.541^{\text {** }}$ \\
\hline $\mathrm{PH}$ & $456186.4^{* *}$ & $604.8^{* *}$ & $1110.47^{*}$ & 303.41 & $469.79^{* *}$ & $294.9^{* *}$ & $314.3^{* *}$ \\
\hline NOB & $0.46^{* *}$ & $0.32^{* *}$ & $0.59^{*}$ & 0.34 & $0.23^{* *}$ & 0.052 & 0.043 \\
\hline $\mathrm{NF}$ & $4334.2^{* *}$ & $24.31^{* *}$ & $45.19^{*}$ & 24.92 & $17.36^{* *}$ & $8.41^{* * *}$ & $7.96^{* *}$ \\
\hline FL & $568.8^{* *}$ & $2.33^{* * *}$ & 2.06 & 3.20 & $2.33^{* *}$ & $0.61^{*}$ & $0.61^{*}$ \\
\hline FD & $1.63^{* *}$ & $0.08^{* *}$ & 0.11 & $0.19^{*}$ & $0.05^{\text {** }}$ & 0.01 & 0.01 \\
\hline FW & $430.63^{* *}$ & $1.87^{* *}$ & 2.87 & $4.20^{*}$ & $1.28^{* *}$ & $0.47^{*}$ & $0.50^{*}$ \\
\hline FY & $1073573.0^{* * *}$ & $6956.41^{\text {*** }}$ & $12204.37^{*}$ & 9416.2 & $4933.77^{* *}$ & $1946.0^{* *}$ & $1806.4^{* *}$ \\
\hline SL & $0.44^{* *}$ & $0.07^{* *}$ & $0.14^{*}$ & 0.09 & $0.05^{*}$ & $0.04^{*}$ & $0.03^{*}$ \\
\hline $\mathrm{CF}$ & $4.85^{* *}$ & $0.88^{* *}$ & 1.01 & 0.64 & $0.87^{* *}$ & $0.41^{\text {** }}$ & $0.36^{\text {** }}$ \\
\hline SLD & $21.13^{* *}$ & $1.96^{* *}$ & 3.07 & 0.96 & $1.71^{* * *}$ & $0.05^{* *}$ & $0.05^{* *}$ \\
\hline
\end{tabular}

and ${ }^{* *}$ Significant at $P=0.05$ and 0.01 probability level, respectively 
Table.4 Estimates of various components of the total variance for the yield components of the fourteen parents and their forty $\mathrm{F}_{1}$ hybrids of okra across environments

\begin{tabular}{|c|c|c|c|c|c|c|c|c|c|c|c|c|c|}
\hline & \multirow{2}{*}{\multicolumn{13}{|c|}{ Traits }} \\
\hline \multirow{2}{*}{ Estimates } & & & & & & & & & & & & & \\
\hline & DFF & FFN & IL & $\mathrm{PH}$ & NOB & $\mathrm{NF}$ & FL & FD & FW & FY & SL & $\mathrm{CF}$ & SLD \\
\hline$\sigma^{2} \mathrm{e}$ & $7.815^{* *}$ & $0.729^{* * *}$ & $7.893^{* * *}$ & $2815.129^{* *}$ & $0.0026^{* * *}$ & $26.737^{* * \pi}$ & $3.506^{* * *}$ & $0.010^{* * *}$ & $2.655^{* * 4}$ & $6625.005^{* *}$ & $0.0026^{*}$ & $0.029^{*}$ & $0.130^{* * *}$ \\
\hline$\sigma^{2} \mathrm{gca}$ & 0.469 & 0.009 & $0.119^{*}$ & $9.071^{* *}$ & $0.006^{* *}$ & $0.507^{*}$ & 0.029 & $0.002^{*}$ & $0.047^{*}$ & $166.487^{* *}$ & $0.0014^{*}$ & 0.012 & 0.031 \\
\hline$\sigma^{2} \mathrm{sca}$ & $1.640 *$ & $0.086^{*}$ & $0.377^{*}$ & $37.149^{* *}$ & $0.021^{* *}$ & $1.618^{* *}$ & $0.172^{* *}$ & $0.004^{* *}$ & 0.083 & $512.467^{* *}$ & 0.0025 & $0.091^{* *}$ & $0.186^{* *}$ \\
\hline$\sigma^{2} \mathrm{gca} / \sigma^{2} \mathrm{sca}$ & 0.285 & 0.104 & 0.315 & 0.244 & 0.285 & 0.313 & 0.168 & 0.500 & 0.566 & 0.324 & 0.560 & 0.131 & 0.166 \\
\hline $\mathrm{h}_{\mathrm{ns}}^{2}(\%)$ & 15.43 & 4.19 & 15.50 & 13.25 & 31.46 & 19.52 & 25.02 & 39.47 & 46.33 & 21.68 & 20.57 & 8.84 & 23.96 \\
\hline GAM (\%) & 1.69 & 0.93 & 4.49 & 2.31 & 30.42 & 5.61 & 2.26 & 5.92 & 3.97 & 9.05 & 2.09 & 1.88 & 7.27 \\
\hline Error & 6.847 & 0.246 & 0.763 & 135.451 & 0.041 & 2.797 & 0.781 & 0.015 & 0.540 & 321.56 & 0.030 & 0.048 & 0.033 \\
\hline
\end{tabular}

$\sigma^{2}$ gca $=$ General combining ability variance.

$\sigma^{2}$ sca $=$ Specific combining ability variance.

$\sigma^{2} \mathrm{e}=$ Environmental variance

" and ${ }^{* *}$ Significant at $P=0.05$ and 0.01 probability level, respectively

Table.5 Estimates of general combining ability for the studied traits of the fourteen parents of okra over pooled environments

\begin{tabular}{|c|c|c|c|c|c|c|c|c|c|c|c|c|c|}
\hline \multirow{2}{*}{ Parents } & \multicolumn{13}{|c|}{ Traits } \\
\hline & DFF & FFN & IL & $\mathrm{PH}$ & NOB & $\mathrm{NF}$ & FL & FD & FW & FY & SL & $\mathrm{CF}$ & SLD \\
\hline \multicolumn{14}{|l|}{ Lines } \\
\hline VIO 44244 & 0.30 & 0.16 & $-0.38^{* *}$ & -3.10 & $-0.13^{* *}$ & $-1.63^{* *}$ & -0.20 & $-0.05^{*}$ & -0.13 & $-21.27^{* *}$ & $-0.06^{*}$ & -0.04 & $0.06^{* *}$ \\
\hline IC -111493 & -0.64 & -0.12 & 0.13 & 3.41 & 0.06 & $0.69^{*}$ & 0.08 & 0.005 & 0.23 & $13.56^{* *}$ & $-0.12^{* *}$ & $-0.10^{* *}$ & $0.53^{* *}$ \\
\hline $\mathrm{JOL}-08-13$ & 0.27 & 0.13 & -0.70 & $-5.75^{* *}$ & 0.03 & $0.70^{*}$ & -0.17 & -0.03 & -0.04 & $8.13^{* *}$ & 0.03 & $0.17^{* *}$ & $0.18^{* * *}$ \\
\hline$E C-284327$ & $2.01^{* *}$ & $0.17^{*}$ & -0.20 & $-7.37^{* * *}$ & $-0.22^{* * *}$ & $-1.23^{* *}$ & -0.06 & $-0.07^{* *}$ & $-0.39^{* *}$ & $-22.18^{* *}$ & $-0.06^{*}$ & $0.16^{* * *}$ & -0.04 \\
\hline IC -045796 & $-0.893^{*}$ & $-0.24^{* *}$ & $0.58^{* * *}$ & $9.52^{* * *}$ & $0.11^{* *}$ & $0.78^{* *}$ & 0.28 & $0.07^{* *}$ & $0.31^{*}$ & $16.45^{* * *}$ & 0.02 & $-0.23^{* *}$ & $0.21^{* * *}$ \\
\hline IC -052273 & -0.77 & 0.05 & 0.15 & -1.13 & -0.03 & -0.12 & -0.09 & 0.001 & -0.01 & -2.88 & $\begin{array}{l}-0.02 \\
-0.02\end{array}$ & $-0.07^{*}$ & $-0.31^{* *}$ \\
\hline JOL $-10-18$ & -0.16 & -0.09 & $-0.32^{*}$ & -0.05 & -0.04 & -0.41 & -0.12 & -0.02 & -0.07 & $-8.18^{* * *}$ & 0.03 & $0.26^{* *}$ & $-0.35^{* *}$ \\
\hline $\mathrm{AOL}-09-17$ & -0.35 & 0.09 & -0.02 & 1.40 & $0.08^{*}$ & 0.28 & 0.10 & 0.004 & -0.10 & 1.37 & 0.04 & $0.11^{* *}$ & $-0.39^{* *}$ \\
\hline VIO 47672 & $-1.42^{* *}$ & -0.14 & $0.70^{* *}$ & $7.45^{* *}$ & $0.23^{* *}$ & $2.01^{* *}$ & $0.48^{\text {** }}$ & $0.12^{* *}$ & $0.52^{* *}$ & $33.91^{* *}$ & $0.08^{* *}$ & $-0.20^{* *}$ & $0.15^{* *}$ \\
\hline $\mathrm{EC}-305623$ & $1.66^{* *}$ & -0.01 & $-0.57^{* *}$ & $-4.38^{*}$ & -0.05 & $-1.04^{* *}$ & -0.28 & -0.02 & $-0.33^{* *}$ & $-18.92^{* *}$ & 0.03 & -0.03 & -0.02 \\
\hline S.E.g $g_{i}$ & 0.61 & 0.12 & 0.20 & 2.74 & 0.04 & 0.39 & 0.20 & 0.03 & 0.17 & 4.22 & 0.04 & 0.05 & 0.04 \\
\hline \multicolumn{14}{|l|}{ Testers } \\
\hline $\mathrm{GAO}-5$ & -0.22 & -0.03 & 0.15 & 0.33 & $0.05^{*}$ & 0.22 & -0.01 & -0.001 & 0.07 & $4.58^{*}$ & $0.04^{*}$ & $0.06^{* * *}$ & $0.05^{* * *}$ \\
\hline GJO - 3 & $-0.69^{*}$ & $-0.13^{*}$ & $0.40^{* *}$ & $2.20^{*}$ & $0.06^{*}$ & $0.57^{* *}$ & $0.26^{* *}$ & $0.06^{* *}$ & $0.28^{* *}$ & $11.58^{* *}$ & -0.001 & $-0.11^{* *}$ & $0.11^{* * *}$ \\
\hline Arka Anamika & 0.37 & 0.06 & $-0.27^{* *}$ & $-2.44^{*}$ & $-0.07^{* *}$ & $-0.64^{* *}$ & $-0.18^{*}$ & -0.03 & $-0.20^{*}$ & $-11.82^{* * *}$ & $-0.04^{*}$ & $0.06^{* *}$ & $-0.11^{* *}$ \\
\hline Varsha Uphar & 0.54 & 0.10 & $-0.29^{* *}$ & 1.97 & -0.04 & -0.15 & -0.06 & $-0.04^{* *}$ & -0.15 & $-4.34^{*}$ & 0.01 & -0.02 & $-0.05^{* *}$ \\
\hline S.E.g $g_{i}$ & 0.39 & 0.07 & 0.13 & 1.73 & 0.03 & 0.25 & 0.13 & 0.02 & 0.11 & 2.67 & 0.02 & 0.03 & 0.02 \\
\hline gi - gj (Line) & 1.21 & 0.23 & 0.40 & 5.40 & 0.09 & 0.77 & 0.41 & 0.05 & 0.34 & 8.32 & 0.08 & 0.10 & 0.08 \\
\hline gi - gj (Tester) & 0.76 & 0.14 & 0.25 & 3.41 & 0.05 & 0.49 & 0.25 & 0.03 & 0.21 & 5.26 & 0.05 & 0.06 & 0.05 \\
\hline
\end{tabular}


Table.6 Promising parents, good general combiners, best performing hybrids and best specific crosses for different traits across the environments

\begin{tabular}{|c|c|c|c|c|c|c|c|c|c|}
\hline \multirow{2}{*}{ Traits } & \multicolumn{2}{|c|}{ Best performing parents } & \multicolumn{2}{|c|}{ Good general combiners } & \multicolumn{3}{|c|}{ Best specific crosses } & \multirow{2}{*}{$S_{i j}-S_{i k}$} & \multirow{2}{*}{$\mathrm{S}_{\mathrm{ij}}-\mathrm{S}_{\mathrm{k}}$} \\
\hline & Parent & Mean & Parent & GCA effect & Cross & SCA effect & Combination & & \\
\hline DFF & Varsha Uphar & 45.61 & VIO 47672 & $-1.42^{* * *}$ & JOL - 08-13 x Arka Anamika & $-1.98^{* * *}$ & Good x Poor & 2.43 & 4.03 \\
\hline FFN & IC-111493 & 4.93 & IC-045796 & $-0.24^{* *}$ & IC - 052273 x Varsha Uphar & $-0.53^{* *}$ & Good x Poor & 0.46 & 0.76 \\
\hline IL & JOL-08-13 & 7.98 & EC-305623 & $-0.57^{* *}$ & $\mathrm{IC}-052273 \times \mathrm{GJO}-3$ & $-1.49^{* *}$ & Good x Poor & 0.81 & 1.34 \\
\hline $\mathrm{PH}$ & VIO 47672 & 135.47 & IC -045796 & $9.52^{* *}$ & VIO 47672 x GJO - 3 & $18.28^{* *}$ & Good x Good & 10.80 & 17.92 \\
\hline NOB & JOL-08-13 & 2.47 & VIO 47672 & $0.23^{* *}$ & VIO 44244 x Varsha Uphar & $0.29^{* *}$ & Poor x Poor & 0.18 & 0.31 \\
\hline NF & IC-045796 & 17.28 & VIO 47672 & $2.01^{* *}$ & $\mathrm{IC}-111493 \times \mathrm{GJO}-3$ & $2.10^{* *}$ & Good x Good & 1.55 & 2.57 \\
\hline FL & Arka Anamika & 11.15 & VIO 47672 & $0.48^{* *}$ & JOL - $08-13 \times$ Arka Anamika & $0.73^{*}$ & Poor x Poor & 0.82 & 1.36 \\
\hline FD & $\begin{array}{l}\text { 1. VIO } 47672 \\
\text { 2. GAO }-5\end{array}$ & 1.56 & VIO 47672 & $0.12^{* *}$ & $\begin{array}{l}\text { 1. JOL }-08-13 \text { x Arka } \\
\text { Anamika } \\
\text { 2. IC }-111493 \times \text { GJO }-3\end{array}$ & $0.13^{* *}$ & $\begin{array}{l}\text { 1. Poor x Poor } \\
\text { 2. Average x Good }\end{array}$ & 0.11 & 0.19 \\
\hline FW & IC-111493 & 10.89 & VIO 47672 & $0.52^{* *}$ & $\begin{array}{l}\text { 1. IC }-111493 \times \text { GJO }-3 \\
\text { 2. JOL }-10-18 \times \text { Varsha Uphar }\end{array}$ & $0.58^{* *}$ & $\begin{array}{l}\text { 1. Average x Good } \\
\text { 2. Poor x Poor }\end{array}$ & 0.68 & 1.13 \\
\hline FY & GAO-5 & 204.98 & VIO 47672 & $33.91^{* *}$ & VIO 47672 x GJO - 3 & $36.00^{* *}$ & Good x Good & 16.65 & 27.61 \\
\hline SL & EC-284327 & 2.23 & IC- 111493 & $-0.12^{* *}$ & VIO 44244 x Arka Anamika & $-0.11^{*}$ & Good x Good & 0.16 & 0.27 \\
\hline $\mathrm{CF}$ & EC-284327 & 4.22 & IC-045796 & $-0.23^{* *}$ & VIO 44244 x GJO - 3 & $-0.48^{* *}$ & Average x Good & 0.20 & 0.33 \\
\hline SLD & GAO-5 & 3.96 & IC-111493 & $0.53^{* *}$ & VIO 47672 x Arka Anamika & $0.70^{* *}$ & Good x Poor & 0.16 & 0.28 \\
\hline
\end{tabular}

Sij - Sik: Difference between two SCA of two hybrids, with a common parent. (L.S.D.0.05)

$\mathrm{Sij}$ - Skl: Difference between two SCA of two hybrids, with non-common parent. (L.S.D.0.05)

and ${ }^{* *}$ Significant at $P=0.05$ and 0.01 probability level, respectively

Table.7 Promising heterotic crosses and their performance for fruit yield per plant and other related parameters

\begin{tabular}{|c|c|c|c|c|c|c|}
\hline \multirow[b]{2}{*}{ Hybrids } & \multirow{2}{*}{$\begin{array}{l}\text { Fruit yield } \\
\text { per Plant }(g)\end{array}$} & \multirow{2}{*}{$\begin{array}{l}\text { SCA } \\
\text { effect }\end{array}$} & \multicolumn{2}{|c|}{ GCA effect } & \multirow{2}{*}{$\begin{array}{l}\text { Standard } \\
\text { Heterosis } \\
(\%)\end{array}$} & \multirow{2}{*}{$\begin{array}{l}\text { Yield attributing traits having significant } \\
\text { and desirable heterosis over standard check }\end{array}$} \\
\hline & & & $\mathrm{P}_{1}$ & $\mathrm{P}_{2}$ & & \\
\hline VIO $47672 \times$ GJO - 3 & 278.01 & $1.77^{* *}$ & $33.91^{* * *}$ & $11.58^{* * *}$ & $33.33^{* *}$ & $\mathrm{NB}, \mathrm{NF}, \mathrm{DFF}, \mathrm{PH}, \mathrm{FL}, \mathrm{FD}, \mathrm{FW}$ \\
\hline IC $-045796 \times$ GJO -3 & 256.16 & $2.10^{* *}$ & $16.45^{* *}$ & $11.58^{* *}$ & $22.83^{* *}$ & $\mathrm{NB}, \mathrm{NF}, \mathrm{FL}, \mathrm{FD}, \mathrm{FW}$ \\
\hline $\mathrm{IC}-111493 \times \mathrm{GJO}-3$ & 254.99 & $2.10^{* *}$ & $13.56^{* *}$ & $11.58^{* *}$ & $22.27^{* *}$ & NB, NF, DFF, FFN, PH, FL, FD, FW, SL \\
\hline $\mathrm{IC}-045796 \times \mathrm{GAO}-5$ & 249.26 & $1.68^{* *}$ & $16.45^{* *}$ & $4.58^{* * *}$ & $19.52^{* *}$ & NB, NF, DFF, FL, FD, FW \\
\hline VIO $47672 \times$ GAO - 5 & 241.57 & 0.12 & $33.91^{* *}$ & $4.58^{* *}$ & $15.83^{* *}$ & $\mathrm{NB}, \mathrm{NF}, \mathrm{DFF}, \mathrm{PH}, \mathrm{FL}, \mathrm{FD}, \mathrm{FW}$ \\
\hline
\end{tabular}

${ }^{*}$ and ${ }^{* *}$ Significant at $\mathrm{P}=0.05$ and 0.01 probability level, respectively 
There is always a very strong and positive association of fruit yield with number of fruits, fruit length, fruit weight and fruit diameter. Highest and significant SCA effects were registered by IC -111493 x GJO -3 for number of fruits (2.10), JOL-08-13 x Arka Anamika for fruit length (0.73), JOL-08-13 x Arka Anamika and IC-111493 x GJO-3 for fruit diameter (0.13), IC-111493 x GJO-3 and JOL-10-18 x varsha uphar for fruit weight (0.58), VIO-47672 x GJO-3 for fruit yield (36.00), respectively. Das et al., (2013), Akotkar et al., (2014), Katagi et al., (2015) and Wakode et al., (2016) also reported similar results.

The estimates of GCA effects revealed that the parental lines showing high GCA effects for fruit yield per plant also exhibited high to average GCA effects for majority of its yield components. It was also interesting to note that involvement of parents with good GCA effects had resulted into hybrids expressing useful heterosis for various traits in majority of cases. The crosses exhibiting desirable and significant SCA and heterosis effects for fruit yield were also associated with high and favourable SCA effects for multiple yield contributing traits. The highest fruit yielding hybrid VIO 47672 x GJO - 3 (Good x Good) had significant desirable sca effect and standard heterosis as well for fruit yield and many of the yield components followed by the cross, IC -111493 x GJO - 3 (Table 7). Corresponding to these findings, Das et al., (2013) reported that positive sca effects were discernible in the hybrids involving both the parents possessing significant and positive GCA effects. The heterotic effects observed for fruit yield might have resulted from interaction of dominant genes contributed by both the parents. Hybrids involving both the parents possessing significant and positive GCA effects (good $x$ good) with higher significant SCA effects for number of fruits per plant, fruit yield per plant and total fruit yield in okra have been reported by Aulakh et al., (2012), Akotkar et al., (2014) and Katagi et al., (2015).

In conclusion, combining ability and heterosis estimates are potential tool for the enhancement of the breeding program in okra. On the basis of combining ability and heterosis estimates, hybrid VIO 47672 x GJO -3 was identified as the best hybrid for fruit yield and its attributes. For fruit yield and its attributes, line VIO 47672 and tester GJO - 3 were the best combiners across the environments.

\section{Abbreviations}

D.F. = degree of freedom, $\mathrm{GA}=$ genetic advance, $\mathrm{DFF}=$ days to first flowering, $\mathrm{FFN}=$ first flowering node, $\mathrm{IL}=$ internodal length, $\mathrm{PH}=$ plant height, $\mathrm{NOB}=$ number of branches, $\mathrm{NF}=$ number of fruits, $\mathrm{FL}=$ fruit length, $\mathrm{FD}=$ fruit diameter, $\mathrm{FW}=$ fruit weight, $\mathrm{FY}=$ fruit yield, $\mathrm{SL}=$ stalk length, $\mathrm{CF}=$ crude fibre content and $\mathrm{SLD}=$ shelf life days, $\mathrm{SCA} / \mathrm{sca}=$ specific combining effects, GCA/gca = general combining effects

\section{Acknowledgement}

The author is thankful to the Department of Science \& Technology, Govt. of India for felicitating INSPIRE fellowship for financial support.

\section{References}

Akotkar P. K., De D. K. and Dubey U. 2014. Genetic studies on fruit yield and yield attributes of okra (Abelmoschus esculentus (L.) Moench). Electron. J. Plant Breed. 5(1): $38-44$.

Anonymous. 2016. Vegetable Varieties developed by IIVR.

Aulakh P.S., Dhall R.K. and Singh J. 2012. Genetics of early and total yield in okra (Abelmoschus esculentus (L.) Moench). Veg. Sci. 39: $165-168$.

Badiger M., Pitchaimuthu M., Parvati, B. and Sachin U. 2014. Heterosis studies in 
conventional and genetic male sterility based hybrids in okra (Abelmoschus esculentus). Green Farming 5(4): 524 - 528.

Dabhi K.H., Vachhani J.H., Poshiya V.K., Jivani L.L. and Kachhadia V.H. 2010. Combining ability for fruit yield and its components over environments in okra [Abelmoschus esculentus (L.) Moench]. Research on Crops 11: $383-390$.

Das S., Chattopadhyay A., Dutta S., Chattopadhyay S. And Hazra P. 2013. Breeding okra for higher productivity and yellow vein mosaic tolerance. International Journal of Vegetable Science 19: 58 - 77.

FAOSTAT. 2013. Production - Crops data. Food and Agriculture Organization of the United Nations. http://www.fao.org/faostat.

Flemine Xavier, Raj Kumar, Behera T. K. and Yadav, R. K. 2016. Studies on heterosis for qualitative traits in okra. Indian J. Hortic. 73(4): $532-537$.

Johnson R.W., Robinson H.F. and Comstock R.E. 1955. Estimating genetic and environmental variability in soya bean. Agron. J. 47: 314318.

Katagi A., Tirakannanvar S. and Jagadeesha R.C. 2015: Combining ability through diallel analysis in okra [Abelmoschus esculentus (L.) Moench]. Green Farming 6: 26 - 29.

Kempthrone O. 1957. An Introduction to Genetics Statistics. John Willey and Sons, New York, pp. $453-471$.

Kumar S., Singh A.K., Das R., Datta S., Arya, K. 2014. Combining ability and its relationship with gene action in okra [Abelmoschus esculentus (L.) Meonch]. J. Crop Weed 10: $82-92$.

Lyngdoh R., Mulge R. and Shadap, A. 2013. Heterosis and combining ability studies in near homozygous lines of okra [Abelmoschus esculentus (L.) Moench] for growth parameters. The Bioscan 8: 1275 - 1279.
Maciel G.M., Luz J.M.Q., Campos S.F.B., Finzi R.R. and Azevedo B.N.R. 2017. Heterosis in okra hybrids obtained by hybridization of two methods: traditional and experimental. Hortic. bras. 35: 119-123. DOI http://dx.doi.org/10.1590/S0102-053620170 118

Obiadalla Ali, Eldekashi M.H. and Helaly A.A. 2013. Combining ability and heterosis studies for yield and its components in some cultivars of okra. Amer-Eurasian J. Agri. \& Environ. Sciences 13: 162 - 167.

Panse V.G. and Sukhatme P.V. 1967. Statistical Methods for Agricultural Workers. Indian Council of Agricultural Research, New Delhi, India, pp. $152-161$.

Robinson H.F., Comstock R.E. and Harvey P.H. 1955. Estimates of heritability and the degree of dominance in maize. Agron. J. 41: 353359.

Singh B., Aakansha Goswami and Sharma A.K. 2016. Estimation of heterosis in okra (Abelmoschus esculentus) for fruit yield and its components using line $\times$ tester mating design. Indian J. Agr. Sci. 86: 1613-1620.

Solankey S.S., Singh A.K. and Singh R.K. 2016. Heterosis of okra resistance sources for okra yellow vein mosaic virus (OYVMV) in okra (Abelmoschus esculentus). Indian J. Agr. Sci. 86: 1460-1465.

Thimmaiah S.R. 1999. Standard methods of biochemical analysis. Kalyani publishers, New Delhi, pp. $64-65$.

Wakode M.M., Bhave S.G., Navhale V.C., Dalvi V.V., Devmore J.P. and Mahadik S.G. 2016. Combining ability studies in okra [Abelmoschus esculentus L. Moench]. Electron. J. Plant Breed. 7: 1007-1013. DOI: 10.5958/0975-928X.2016.00138

\section{How to cite this article:}

Sanket J. More, K.N. Chaudhari, G.B. Vaidya and Chawla, S.L. 2017. Multi-Environment Analyses of Genetic Components and Combining Abilities in Relation to Heterosis in Okra [Abelmoschus esculentus (L.) Moench]. Int.J.Curr.Microbiol.App.Sci. 6(12): 2835-2842. doi: https://doi.org/10.20546/ijcmas.2017.612.330 